\title{
On the Existence of Luminosity Differences between HB Field and Cluster Stars
}

\author{
E. Carretta, R. G. Gratton \\ Osservatorio Astronomico di Padova, vicolo dell'Osservatorio 5, I-35122 \\ Padova, Italy \\ G. Clementini \\ Osservatorio Astronomico di Bologna, via Ranzani 1, I-40127 Bologna, \\ Italy
}

\begin{abstract}
The discrepancy between the long distance scale as derived, e.g., from Hipparcos-based distances to globular clusters via main sequence fitting to local subdwarfs, and the short distance scale as derived, e.g., from the absolute magnitude of field RR Lyrae stars via statistical parallaxes and the Baade-Wesselink method, could be accounted for if an intrinsic difference in luminosity of about $0.1-0.2 \mathrm{mag}$ were found to exist between horizontal branch (HB) stars populating the sparse general field and the dense globular clusters.

We discuss the possible existence of such a systematic difference, comparing the period-shifts observed for field and cluster RR Lyrae stars. Various approaches based on different parameters and datasets for both cluster and field variables are used in order to establish the size of such a hypothetical difference, if any.

We find that very small, not significant differences do exist between the period-metallicity distributions of field and cluster RR Lyrae stars, thus confirming, with a more quantitative approach, the qualitative conclusions by Catelan (1998).

This observational evidence translates into an almost negligible difference between the HB luminosity of field and cluster stars, unless RR Lyrae stars in globular clusters are about $0.06 M_{\odot}$ more massive than field RR Lyrae stars at the same metallicity, which is still to be proven.
\end{abstract}

\section{Reference}

Catelan, M. 1998, ApJ, 495, L81 